\title{
Analysis of geometric factors for an X-ray fluorescence analyzer designed via the Monte Carlo method
}

\author{
LIU Hefan ${ }^{1, a}$, TIAN Hong ${ }^{1}$, ZHOU Laidong ${ }^{1}$, ZHOU Zihang $^{1}$, HUANG Fengxia ${ }^{1}$, LU Chengwei $^{1}$, ZENG Guogiang ${ }^{2}$ and HU \\ Xiang $^{1}$ \\ ${ }^{1}$ Chengdu Research Academy of Environmental Sciences, Chengdu 610072, China \\ ${ }^{2}$ Chengdu University of Technology, Sichuan, Chengdu, 610059, China
}

\begin{abstract}
This paper presents a theoretical study on the design of an X-ray fluorescence (XRF) analyzer. The relative geometric positions of the analyzer's source, detector and specimen are emphasized, which impacts the analyte's characteristic X-ray fluorescence counts. The theoretical formula for the X-ray fluorescence intensity was derived. The geometric factors (angle and distance) were simulated using Monte Carlo Neutron-Particle Transport Code MCNP5. The Cu's X-ray characteristic peak counts were calculated. These Monte Carlo model calculations analyzed two types of geometry changes. The best geometric positions for the XRF analyzer had the incident angle for the excitation source $(\alpha)$ equivalent to the exit angle for the specimen's characteristic X-ray ( $\beta$ ). The maximal characteristic X-ray peak counts were obtained when $\alpha$ and $\beta$ were orthogonal, and the minimum counts were obtained when parallel. To increase the fluorescence counts, the source and detector should be set as close to the specimen as possible. This method and these conclusions can provide technical guidance for designing XRF analyzers. Key words: Geometric factors; X-ray fluorescence analyzer; Monte Carlo method; MCNP code
\end{abstract}

\section{Instruction}

$\mathrm{X}$-ray fluorescence (XRF) analyzers are one of the most important analytical instrument types for element analyses [1-6]. The analyte's characteristic X-ray fluorescence counts relate not only to its physical and chemical properties but also the geometric positions of the source, specimen and detector [7-9]. The XRF analyzer's geometric setup can change the detector's excitation area, detection area and X-ray absorption by the surrounding medium. The best geometric conditions yield the maximal counts, highest analytical accuracy, and minimal measuring time. Many methods are used to find the optimal geometric conditions when designing XRF analyzers. The Monte Carlo Neutron-Particle Transport Code MCNP5, produced by Los Alamos National Laboratory, is important software for nuclear analysis including simulating and calculating XRF spectroscopy [10]. With relative errors below 5\%, the spectral intensity accuracy predicted by the MCNP5 code is approximately $95 \%$, and the prediction accuracy ranges from $90 \%$ to $97 \%$ for unknown specimen compositions [11]. Several models were established in this paper using the MCNP5 code for XRF analyzers. The best geometric conditions were obtained by varying the geometric parameters for the XRF analyzer design via the Monte Carlo method.

\section{Theoretical calculations}

The basic hypotheses follow: the specimen surface is smooth; the elements in the analyte are evenly distributed; the excitation source is monoenergetic; the incident X-ray beam is parallel to the analyte's characteristic X-ray beam. According to the Beer-Lambert Law, the original incident X-ray fluorescence intensity of the specimen is:

$$
I_{0}^{\prime}=I_{0} \exp \left[-\mu\left(S, \lambda_{0}\right) \rho x \cos \alpha\right]
$$

In Eq. (1), $I_{0}$ is the incident X-ray fluorescence intensity in primary-sectional units. $\mu\left(S, \lambda_{0}\right)$ is the combined mass absorption coefficient. $S$ is the analyte. $\lambda_{0}$ is the incident X-ray wavelength. $\rho$ is the specimen's density. $\alpha$ is the incident angle between the incident Xray beam and specimen surface.

If $i$ elements are uniformly distributed throughout the specimen, $C_{i}$ is its content, and $I_{x}$ is the analyte's characteristic X-ray fluorescence intensity. Therefore, the characteristic X-ray intensity from a $d x$ layer is as follows:

$$
d I_{x}=I_{0}^{\prime} C_{i} \mu\left(i, \lambda_{i}\right) \rho \cos \alpha E_{i} d x
$$

In Eq. (2), element $i$ is excited by incident X-rays of wavelength $\lambda_{i} ; \mu\left(i, \lambda_{i}\right)$ is the absorption coefficient quality; $E_{i}$ is the probability for characteristic X-ray emission when excited by an incident X-ray with wavelength $\lambda_{i}$.

\footnotetext{
$\overline{{ }^{a} \text { Corresponding author: liuhefan@ } 126 . c o m}$
} 
Characteristic X-rays are released uniformly from all directions, and only recorded within the solid angle of the detector. $\beta$ is the exit angle for the X-ray characteristic beam from the specimen's surface. The crossing distance for the characteristic $\mathrm{X}$-ray is $x \cos \beta$ based on the direction for the $d x$ layer from the specimen to the detector. Therefore, the characteristic X-ray fluorescence intensity is as follows:

$$
d I_{i}=\frac{d \Omega}{4 \pi} \exp \left[-\mu\left(S, \lambda_{i}\right) \rho x \cos \beta\right] d I_{x}
$$

Inserting Eq. (1) and Eq. (2) into Eq. (3) yields the following:

$$
\begin{gathered}
d I_{i}=\frac{d \Omega}{4 \pi} I_{0} C_{i} E_{i} \mu\left(i, \lambda_{0}\right) \rho \cos \alpha \exp \left[-\mu\left(S, \lambda_{0}\right) \rho x \cos \alpha\right. \\
\left.-\mu\left(S, \lambda_{i}\right) \rho x \cos \beta\right] d x
\end{gathered}
$$

Integrating Eq. (4) over the thicknesses, $x$, from $x=0$ to $x \rightarrow \infty$ yields:

$$
I_{i}=\frac{d \Omega}{4 \pi} \frac{I_{0} C_{i} E_{i} \mu\left(i, \lambda_{0}\right) \rho \cos \alpha}{\mu\left(S, \lambda_{0}\right) \rho \cos \alpha+\mu\left(S, \lambda_{i}\right) \rho \cos \beta}
$$

In Eq. (5), $I_{i}$ and $C_{i}$ relate to not only the specimen's density, type, mass absorption coefficient, etc. but also the geometric layout for the XRF analyzer, such as the incidence angle, exit angle, detector's solid angle, and so on.

\section{Monte Carlo model descriptions}

\subsection{Basic model}

Many models to determine the influence of different geometric factors and XRF fluorescence counts have been established in this paper. The basic model is shown in Fig. 1.

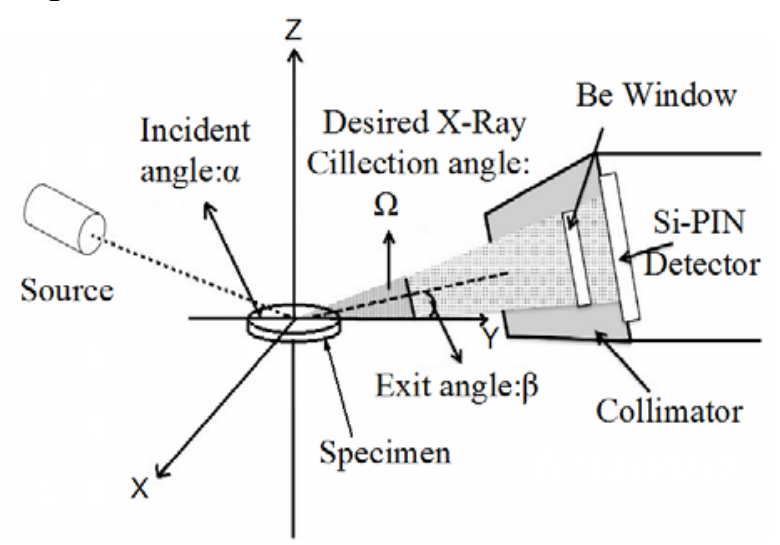

Fig. 1 The geometric layout for designing an X-ray fluorescence measurement system via the Monte Carlo method

In Fig. 1, the model's origin is centered in the specimen's plane. The Y-coordinate is parallel to the specimen, and the Z-coordinate is vertical to the specimen. This model is described in detail in Section 2.2 and the Section 2.3.

\subsection{Angle descriptions}

There are two methods for describing the angle with a Monte Carlo model. The distance between the excitation source and specimen $\left(H_{S-S}\right)$ is $20 \mathrm{~mm}$, so only the distance between the detector and specimen $\left(H_{D-S}\right)$ varies.

(a) The value of $\alpha$ and $\beta$ are simultaneously changed from $0^{\circ}$ to $90^{\circ}, \alpha=\beta$;

(b) Set $\beta=45^{\circ}$ while changing $\alpha$ from $0^{\circ}$ to $180^{\circ}$.

Because the incident $\mathrm{X}$-ray is primarily a diverging cone, the excitation source has a scattering angle. For instance, the scattering angle for an X-ray tube, TUB00083, produced by Moxtek Inc. is $23^{\circ}$. Therefore, $\alpha$ and $\beta$ in Fig. 1 are averages.

\subsection{Distance descriptions}

There are three ways to vary the distance using these Monte Carlo models.

(a) When $H_{D-S}=20 \mathrm{~mm}$ and $\alpha=\beta=45^{\circ}, H_{S-S}$ is changed from $0.1 \mathrm{~mm}$ to $10^{4} \mathrm{~mm}$;

(b) When $H_{S-S}=20 \mathrm{~mm}$ and $\alpha=\beta=45^{\circ}, H_{D-S}$ is changed from $0.1 \mathrm{~mm}$ to $100 \mathrm{~mm}$;

(c) When $H_{S-S}=H_{D-S}=20 \mathrm{~mm}$ and $\alpha=\beta=45^{\circ}$, the distance between the excitation source and detector $\left(H_{S-D}\right)$ is changed from $0.001 \mathrm{~mm}$ to $100 \mathrm{~mm}$.

\section{Discussion}

\subsection{Impact of the angle}

\subsubsection{Simultaneously changing $\alpha$ and $\beta$ from $0^{\circ}$ to $90^{\circ}(\alpha=\beta)$}

Under these conditions, the characteristic X-ray peak counts for $\mathrm{CuK}_{\alpha}$ and $\mathrm{CuK}_{\beta}$ are nearly constant, as shown in Fig. 2.

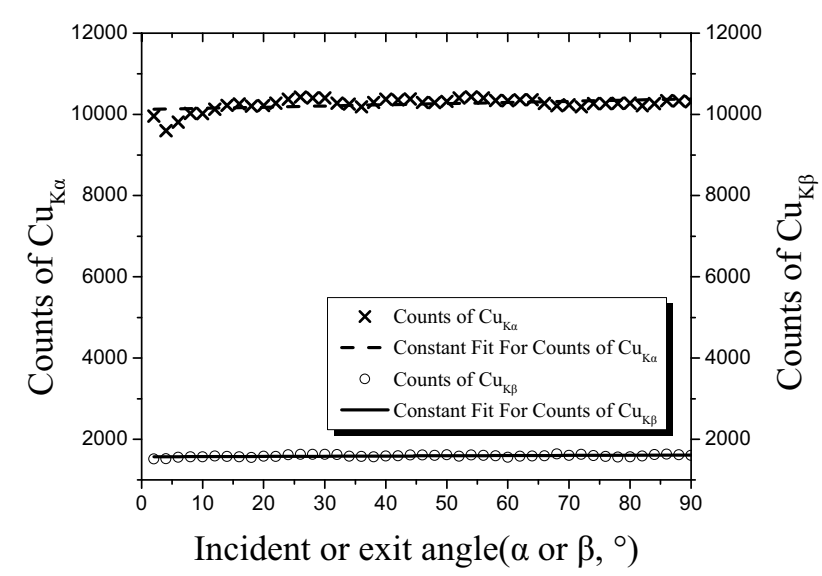

Fig. 2 Relationship between Cu's characteristic X-ray peak counts and the angle from the Monte Carlo calculations $\left(H_{S-S}=H_{D-S}=20 \mathrm{~mm}, \alpha=\beta\right)$ 
In Fig. 2, changing $\alpha$ and $\beta$ has no effect on the measurement results. This conclusion can guide XRF analyzer design.

\subsection{2 $\beta=45^{\circ}$ while changing $\alpha$ from $0^{\circ}$ to $180^{\circ}(\alpha \neq \beta)$}

Under these conditions, the relationship between the angle and characteristic X-ray peak counts for $\mathrm{CuK}_{\alpha}$ and $\mathrm{CuK}_{\beta}$ is shown in Fig. 3.

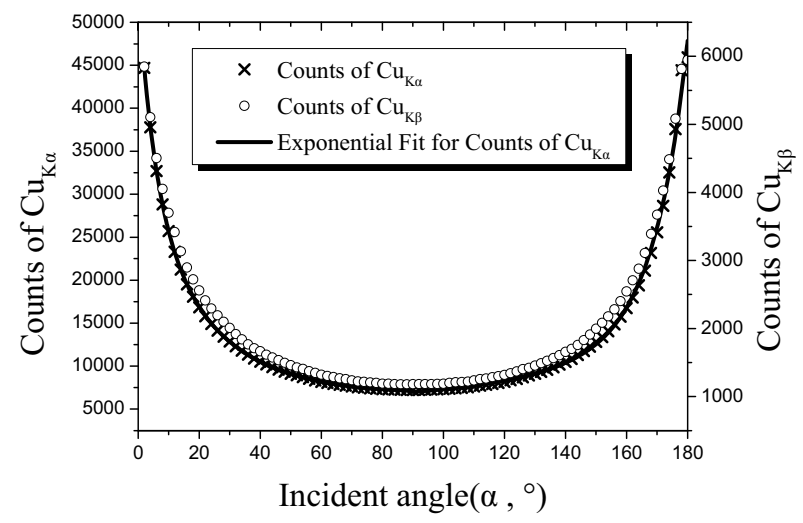

Fig. 3 Relationship between the Cu's characteristic X-ray peak counts and angle from the Monte Carlo calculations

$$
\left(H_{S-S}=H_{D-S}=20 \mathrm{~mm}, \beta=45^{\circ}, \alpha \neq \beta\right)
$$

In Fig. 3, when $\alpha$ and $\beta$ are orthogonal, the characteristic X-ray peak counts for $\mathrm{CuK}_{\alpha}$ and $\mathrm{CuK}_{\beta}$ are maximized; the counts become minimum when they are parallel.

This result occurs because the excitation source and detector are close and even overlap when $\alpha$ and $\beta$ are orthogonal. In this case, $\alpha$ and $\beta$ are at nearly a rightangle, i.e., $\sin \alpha \approx \sin \beta \approx 1$. The probability for the characteristic $\mathrm{X}$-rays to enter the detection window is maximized. Interference due to incident X-rays from the source is minimized. The probability characteristic Xrays are excited by the incident $\mathrm{X}$-rays is maximized. Therefore, the X-ray characteristic peak counts for $\mathrm{CuK}_{\alpha}$ and $\mathrm{CuK}_{\beta}$ are maximal. Similarly, the incident X-ray counts recorded by the detector are maximized when $\alpha$ and $\beta$ are parallel. The specimen's fluorescence probability decreases in the detector's solid angle. The probability the characteristic X-rays are excited by the source decreases. The background counts from scattering increases. In this case, multiple scatterings from the incident and characteristic X-rays further reduce the characteristic X-ray peak counts. Therefore, the characteristic X-ray peak counts for $\mathrm{CuK}_{\alpha}$ and $\mathrm{CuK}_{\beta}$ are minimized.

These results confirm the reasons center or cyclic excitation sources are used for XRF analyzers in practice.

\subsection{Impact of distance}

\subsubsection{Distance between the specimen and excitation source $\left(H_{S-S}\right)$}

In Fig. $4, H_{D-S}$ remains constant, $H_{S-S}$ is below $20 \mathrm{~mm}$, and the characteristic X-ray peak counts for $\mathrm{CuK}_{\alpha}$ and $\mathrm{CuK}_{\beta}$ remain maximal.

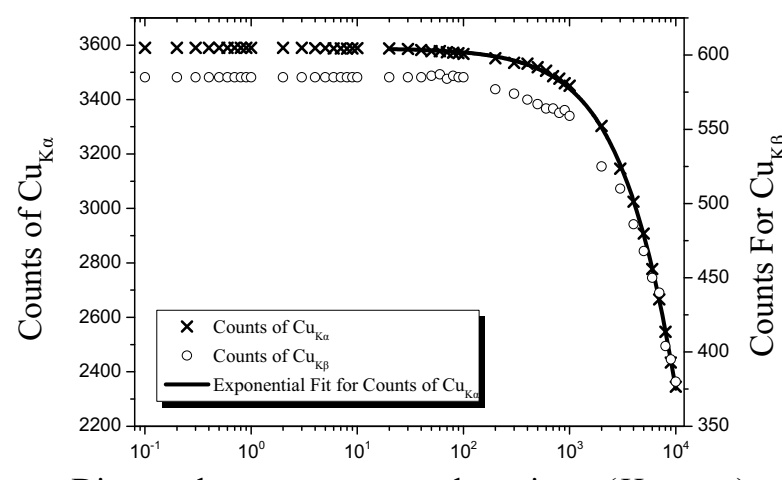

Distance between source and specimen $\left(H_{s-s}, \mathrm{~mm}\right)$

Fig. 4 Relationship between Cu's characteristic X-ray peak counts and $H_{S-S}$ in the Monte Carlo calculations $\left(H_{D-}\right.$ $s=20 \mathrm{~mm}, \alpha=\beta=45^{\circ}$ )

Increasing $H_{S-S}$ decreases the incident X-ray irradiation at the specimen surface. The probability the characteristic X-rays are excited by the source also decreases, which reduces Cu's characteristic X-ray peak counts. In Fig. 4, increasing $H_{S-S}$ further $(\geq 20 \mathrm{~mm})$ exponentially decreases the counts.

\subsubsection{Distance between the detector and specimen $\left(H_{D-S}\right)$}

Holding $H_{S-S}$ constant while increasing $H_{D-S}(<10$ $\mathrm{mm}$ ) exponentially decreased the characteristic X-ray peak counts for $\mathrm{CuK}_{\alpha}$ and $\mathrm{CuK}_{\beta}$. Increasing $H_{D-S}$ further $(\geq 10 \mathrm{~mm})$ reduces the characteristic X-ray peak counts to essentially nothing as shown in Fig. 5.

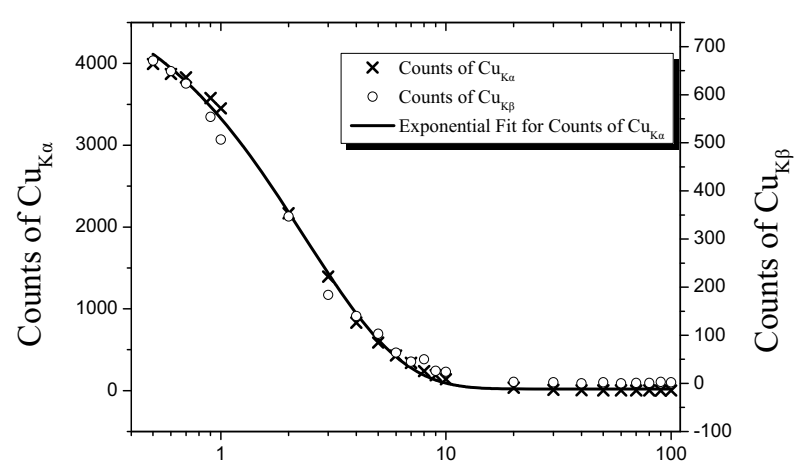

Distance between detector and specimen $\left(H_{D-S}, \mathrm{~mm}\right)$

Fig. 5 Relationship between the Cu's X-ray characteristic peak counts and $H_{D-S}$ in Monte Carlo calculations $\left(H_{S-S}=20\right.$ $\mathrm{mm}, \alpha=\beta=45^{\circ}$ )

Increasing the $H_{D-S}$ decreases the detector's solid angle and Cu's characteristic X-ray peak counts. When $H_{D-S}$ is above $10 \mathrm{~mm}$, the characteristic X-rays are almost outside the solid angle entirely. Therefore, the counts are almost zero. 
Based on Fig. 4 and Fig. 5, the detector and specimen should be as close as possible in the XRF analyzer design to increase the fluorescence counts.

\subsubsection{Distance between the excitation source and detector $\left(H_{S-D}\right)$}

The specimen center is the origin for the coordinate system (in Fig. 1). In this case, the characteristic X-ray peak counts for $\mathrm{CuK}_{\alpha}$ are almost zero when $H_{S-D}$ is below $5 \mathrm{~mm}$. Increasing $H_{S-D}$ also increases the counts. When $H_{S-D}=40 \mathrm{~mm}$, the counts are maximal. Increasing $H_{S-D}$ further $(>40 \mathrm{~mm})$ exponentially decreases the counts.

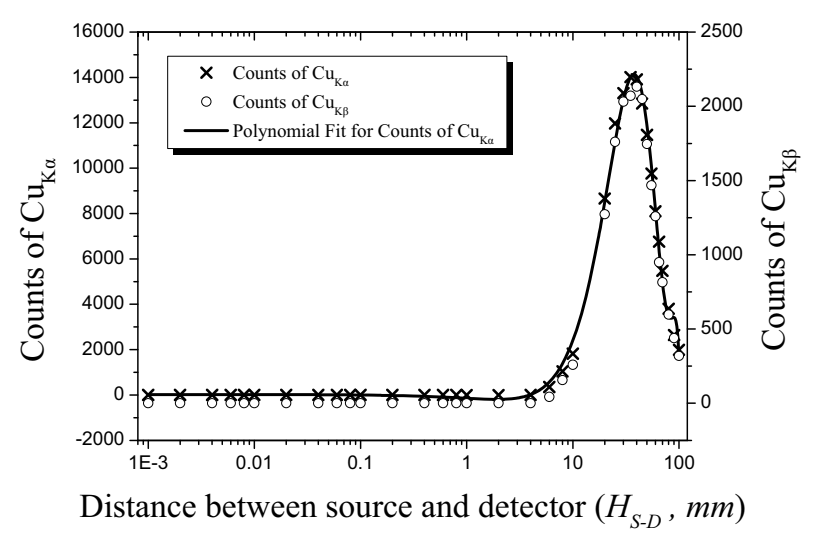

Fig. 6 Relationship between Cu's X-ray characteristic peak counts and $H_{S-D}$ from the Monte Carlo calculations $\left(H_{S-S}=H_{D-S}=20 \mathrm{~mm}, \alpha=\beta=45^{\circ}\right)$

Extremely small or large $H_{S-D}$ values cause the characteristic X-rays to mostly fall outside the detector's solid angle. Therefore, there was essentially no characteristic X-ray peak. There must be a preferred distance for the geometric conditions. In Fig. 6, $H_{S-D}=40$ $\mathrm{mm}$ was the best distance for this paper's model. However, $H_{S-S}=H_{D-S}=20 \mathrm{~mm}$ when $H_{S-D}=40 \mathrm{~mm}$, the relationship between $\alpha$ and $\beta$ is exactly orthogonal. This result is congruent with the conclusions in Section 3.1.2.

\section{Conclusions}

This paper studies the geometric effects for an XRF analyzer designed via the Monte Carlo method. The theoretical formula for the X-ray fluorescence intensity was derived. XRF analysis models were established using the MCNP5 Code. The results were as follows: when $\alpha$ and $\beta$ are simultaneously changed and $\alpha=\beta$, there is no effect on the measured results. There is non-linear relationship between $\alpha$ and the Cu's X-ray characteristic peak counts. The counts are maximized when $\alpha$ and $\beta$ are orthogonal and minimized when $\alpha$ and $\beta$ are parallel. When $H_{D-S}=20 \mathrm{~mm}, \alpha=\beta=45^{\circ}$, the X-ray characteristic fluorescence counts remain constant $\left(H_{S-S}<20 \mathrm{~mm}\right)$. Increasing $H_{S-S}(\geq 20 \mathrm{~mm})$ exponentially decreases the counts. When $H_{S-S}=20 \mathrm{~mm}, \alpha=\beta=45^{\circ}$, and $H_{D-S}$ increases $(<10 \mathrm{~mm})$, the characteristic X-ray peak counts for $\mathrm{CuK}_{\alpha}$ and $\mathrm{CuK}_{\beta}$ decrease exponentially. Increasing $H_{D-S}$ further $(\geq 10 \mathrm{~mm})$ reduces the characteristic X-ray peak counts to nearly zero. This method and these conclusions can provide technical guidance for designing X-ray fluorescence analyzers.

\section{Acknowledgements}

The presented results receive funding from the National High Technology Research and Development Program of China under contract number 2012AA061803. It is also supported by the Special Funds of the National Natural Science Foundation of China (Grant No. 41474159). This work is partly supported by the Opening Fund of Provincial Key Lab of Applied Nuclear Techniques in Geosciences (gnzds2014002, gnzds2014006).

\section{References}

1. Alireza Mazoochi, et al., NUCL INSTRUM METH A. 763 (2014) 538.

2. D. Hampai, et al., Spectrochimica Acta Part B: Atomic Spectroscopy. 101 (2014) 114.

3. P. Oberta, R. Mokso, NUCL INSTRUM METH A. 729 (2013) 85.

4. Graeme M. Hansford, NUCL INSTRUM METH A. 690 (2012) 117.

5. LU Anxiang, et al., SPECTROSC SPECT ANAL 30 (2010) 2848.

6. E G San Miguel, et al., APPL RADIAT ISOTOPES 61 (2004) 361.

7. Tektronix Corporation 2007 TEKVISA Programmer Manual Version1.1071-1101-00.

8. Will J.M., Duncan A.R 2008 Understanding XRF Spectrometry PT-1 7602.

9. Ge Liangquan, Zhang Ye, NUCLEAR TECHNIQUES 18 (1995) 332.

10. Sharshar T., et al., APPL RADIAT ISOTOPES 48 (1997) 695.

11. László Vincze, et al., Spectrochim Acta. 50B (1993) 127. 